PROCEEDINGS OF THE

AMERICAN MATHEMATICAL SOCIETY

Volume 134, Number 12, December 2006, Pages 3465-3474

S 0002-9939(06)08420-6

Article electronically published on June 12, 2006

\title{
UNIVERSAL LOCALIZATION OF TRIANGULAR MATRIX RINGS
}

\author{
DESMOND SHEIHAM
}

(Communicated by Martin Lorenz)

\begin{abstract}
If $R$ is a triangular $2 \times 2$ matrix ring, the columns $P$ and $Q$ are f.g. projective $R$-modules. We describe the universal localization of $R$ which makes invertible an $R$-module morphism $\sigma: P \rightarrow Q$, generalizing a theorem of A. Schofield. We also describe the universal localization of $R$-modules.
\end{abstract}

\section{INTRODUCTION}

Suppose $R$ is an associative ring (with 1) and $\sigma: P \rightarrow Q$ is a morphism between finitely generated projective $R$-modules. There is a universal way to localize $R$ in such a way that $\sigma$ becomes an isomorphism. More precisely, there is a ring morphism $R \rightarrow \sigma^{-1} R$ which is universal for the property that

$$
\sigma^{-1} R \otimes_{R} P \stackrel{1 \otimes \sigma}{\longrightarrow} \sigma^{-1} R \otimes_{R} Q
$$

is an isomorphism (Cohn [7, 9, 8, 6], Bergman [4, 5, Schofield [17]). Although it is often difficult to understand universal localizations when $R$ is non-commutative 1 there are examples where elegant descriptions of $\sigma^{-1} R$ have been possible (e.g. Cohn and Dicks [10, Dicks and Sontag [11, Thm. 24], Farber and Vogel 12 Ara, González-Barroso, Goodearl and Pardo [1, Example 2.5]). The purpose of this paper is to describe and to generalize some particularly interesting examples due to A. Schofield [17, Thm. 13.1] which have application in topology (e.g., Ranicki [16, Part 2]).

We consider a triangular matrix ring $R=\left(\begin{array}{cc}A & M \\ 0 & B\end{array}\right)$ where $A$ and $B$ are associative rings (with 1 ) and $M$ is an $(A, B)$-bimodule. Multiplication in $R$ is given by

$$
\left(\begin{array}{cc}
a & m \\
0 & b
\end{array}\right)\left(\begin{array}{cc}
a^{\prime} & m^{\prime} \\
0 & b^{\prime}
\end{array}\right)=\left(\begin{array}{cc}
a a^{\prime} & a m^{\prime}+m b^{\prime} \\
0 & b b^{\prime}
\end{array}\right)
$$

for all $a, a^{\prime} \in A, m, m^{\prime} \in M$ and $b, b^{\prime} \in B$. The columns $P=\left(\begin{array}{c}A \\ 0\end{array}\right)$ and $Q=\left(\begin{array}{c}M \\ B\end{array}\right)$ are f.g. projective left $R$-modules with

$$
P \oplus Q \cong R \text {. }
$$

Received by the editors October 22, 2004 and, in revised form, May 31, 2005 and July 7, 2005. 2000 Mathematics Subject Classification. Primary 13B30.

Desmond Sheiham died on March 25, 2005. This article was prepared for publication by Andrew Ranicki, with the assistance of Aidan Schofield.

${ }^{1}$ If $R$ is commutative one obtains a ring of fractions; see Bergman [4 p. 68].

(C)2006 American Mathematical Society Reverts to public domain 28 years from publication 3465 
For the general theory of triangular matrix rings see Haghany and Varadarajan [13, 14.

We shall describe in Theorem 2.4 the universal localization $R \rightarrow \sigma^{-1} R$ which makes invertible a morphism $\sigma: P \rightarrow Q$. Such a morphism can be written $\sigma=\left(\begin{array}{l}j \\ 0\end{array}\right)$ where $j: A \rightarrow M$ is a morphism of left $A$-modules. Examples follow, in which restrictions are placed on $A, B, M$ and $\sigma$. In particular, Example 2.8 recovers Theorem 13.1 of Schofield [17. We proceed to describe the universal localization $\sigma^{-1} N=\sigma^{-1} R \otimes_{R} N$ of an arbitrary left module $N$ for the triangular matrix ring $R$ (see Theorem 2).

The structure of this paper is as follows: definitions, statements of results and examples are given in Section 2 and the proofs are collected in Section 3 .

I am grateful to Andrew Ranicki, Aidan Schofield and Amnon Neeman for helpful conversations.

\section{Statements AND EXAMPLES}

Let us first make more explicit the universal property of localization:

Definition 2.1. A ring morphism $R \rightarrow R^{\prime}$ is called $\sigma$-inverting if

$$
\operatorname{id} \otimes \sigma: R^{\prime} \otimes_{R}\left(\begin{array}{c}
A \\
0
\end{array}\right) \rightarrow R^{\prime} \otimes_{R}\left(\begin{array}{c}
M \\
B
\end{array}\right)
$$

is an isomorphism. The universal localization $i_{\sigma}: R \rightarrow \sigma^{-1} R$ is the initial object in the category of $\sigma$-inverting ring morphisms $R \rightarrow R^{\prime}$. In other words, every $\sigma$ inverting ring morphism $R \rightarrow R^{\prime}$ factors uniquely as a composite $R \rightarrow \sigma^{-1} R \rightarrow R^{\prime}$.

Definition 2.2. An $(A, M, B)$-ring $\left(S, f_{A}, f_{M}, f_{B}\right)$ is a ring $S$ together with ring morphisms $f_{A}: A \rightarrow S$ and $f_{B}: B \rightarrow S$ and an $(A, B)$-bimodule morphism $f_{M}: M \rightarrow S$.

$$
A \underset{f_{M}}{\stackrel{f_{A}}{\longrightarrow}} S \stackrel{f_{B}}{\longleftarrow} B
$$

It is understood that the $(A, B)$-bimodule structure on $S$ is induced by $f_{A}$ and $f_{B}$, so that $f_{A}(a) f_{M}(m)=f_{M}(a m)$ and $f_{M}(m) f_{B}(b)=f_{M}(m b)$ for all $a \in A, b \in B$ and $m \in M$.

A morphism $\left(S, f_{A}, f_{M}, f_{B}\right) \rightarrow\left(S^{\prime}, f_{A}^{\prime}, f_{M}^{\prime}, f_{B}^{\prime}\right)$ of $(A, M, B)$-rings is a ring morphism $\theta: S \rightarrow S^{\prime}$ such that i) $\theta f_{A}=f_{A}^{\prime}$, ii) $\theta f_{M}=f_{M}^{\prime}$ and iii) $\theta f_{B}=f_{B}^{\prime}$.

Definition 2.3. Suppose $p \in M$. Let $\left(T(M, p), \rho_{A}, \rho_{M}, \rho_{B}\right)$ denote the initial object in the subcategory of $(A, M, B)$-rings with the property $\rho_{M}(p)=1$. For brevity we often write $T=T(M, p)$.

The ring $T$ can be explicitly described in terms of generators and relations as follows. We have one generator $x_{m}$ for each element $m \in M$ and relations:

(+) $x_{m}+x_{m^{\prime}}=x_{m+m^{\prime}}$,

(a) $x_{a p} x_{m}=x_{a m}$,

(b) $x_{m} x_{p b}=x_{m b}$,

(id) $x_{p}=1$, 
for all $m, m^{\prime} \in M, a \in A$ and $b \in B$. The morphisms $\rho_{A}, \rho_{M}, \rho_{B}$ are

$$
\begin{aligned}
& \rho_{A}: A \rightarrow T ; a \mapsto x_{a p}, \\
& \rho_{B}: B \rightarrow T ; b \mapsto x_{p b}, \\
& \rho_{M}: M \rightarrow T ; m \mapsto x_{m} .
\end{aligned}
$$

Suppose $\sigma:\left(\begin{array}{c}A \\ 0\end{array}\right) \rightarrow\left(\begin{array}{c}M \\ B\end{array}\right)$ is a morphism of left $R$-modules. We may write $\sigma\left(\begin{array}{l}1 \\ 0\end{array}\right)=\left(\begin{array}{l}p \\ 0\end{array}\right)$ for some $p \in M$. Let $T=T(M, p)$.

Theorem 2.4. The universal localization $R \rightarrow \sigma^{-1} R$ is (isomorphic to)

$$
R=\left(\begin{array}{cc}
A & M \\
0 & B
\end{array}\right) \stackrel{\left(\begin{array}{cc}
\rho_{A} & \rho_{M} \\
0 & \rho_{B}
\end{array}\right)}{\longrightarrow}\left(\begin{array}{ll}
T & T \\
T & T
\end{array}\right)
$$

Example 2.5. (1) Suppose $A=B=M$ and multiplication in $A$ defines the $(A, A)$-bimodule structure on $M$. If $p=1$, then $T=A$ and $\rho_{A}=\rho_{M}=$ $\rho_{B}=\mathrm{id}_{A}$.

(2) Suppose $A=B$ and $M=A \oplus A$ with the obvious bimodule structure. If $p=(1,0)$, then $T$ is the polynomial ring $A[x]$ in a central indeterminate $x$. The map $\rho_{A}=\rho_{B}$ is the inclusion of $A$ in $A[x]$ while $\rho_{M}(1,0)=1$ and $\rho_{M}(0,1)=x$.

The universal localizations corresponding to Example 2.5 are

(1) $\left(\begin{array}{cc}A & A \\ 0 & A\end{array}\right) \rightarrow\left(\begin{array}{cc}A & A \\ A & A\end{array}\right)$;

(2) $\left(\begin{array}{cc}A & A \oplus A \\ 0 & A\end{array}\right) \rightarrow\left(\begin{array}{cc}A[x] & A[x] \\ A[x] & A[x]\end{array}\right)$.

Remark 2.6. One can regard the triangular matrix rings in these examples as path algebras over $A$ for the quivers

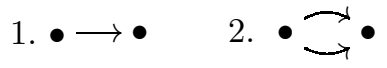

The universal localizations $R \rightarrow \sigma^{-1} R$ are obtained by introducing an inverse to the arrow in (1) and by introducing an inverse to one of the arrows in (2). See for example Benson [2, p. 99] for an introduction to quivers.

The following examples subsume these.

Example 2.7. (1) (Amalgamated free product; Schofield [17, Thm. 4.10]) Suppose $i_{A}: C \rightarrow A$ and $i_{B}: C \rightarrow B$ are ring morphisms and $M=A \otimes_{C} B$. If $p=1 \otimes 1$, then $T$ is the amalgamated free product $A \underset{C}{\sqcup B}$ and appears in the pushout square

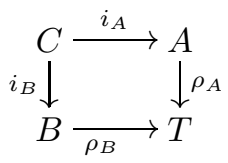

The map $\rho_{M}$ is given by $\rho_{M}(a \otimes b)=\rho_{A}(a) \rho_{B}(b)$ for all $a \in A$ and $b \in B$. We recover part (1) of Example 2.5 by setting $A=B=C$ and $i_{A}=i_{B}=$ id. 
(2) (HNN extension) Suppose $A=B$ and $i_{1}, i_{2}: C \rightarrow A$ are ring morphisms. Let $A \otimes_{C} A$ denote the tensor product with $C$ acting via $i_{1}$ on the first copy of $A$ and by $i_{2}$ on the second copy. Let $M=A \oplus\left(A \otimes_{C} A\right)$ and $p=(1,0 \otimes 0)$. Now $T=A *_{C} \mathbb{Z}[x]$ is generated by the elements in $A$ together with an indeterminate $x$ and has the relations in $A$ together with $i_{1}(c) x=x i_{2}(c)$ for each $c \in C$. We have $\rho_{A}(a)=\rho_{B}(a)=a$ for all $a \in A$ while $\rho_{M}(1,0 \otimes 0)=1$ and $\rho_{M}\left(0, a_{1} \otimes a_{2}\right)=a_{1} x a_{2}$. If $C=A$ and $i_{1}=i_{2}=\mathrm{id}_{A}$, we recover part (2) of Example 2.5.

The following example is Theorem 13.1 of Schofield [17] and generalizes Example 2.7

Example 2.8. (1) Suppose $p$ generates $M$ as a bimodule, i.e., $M=A p B$. Now $T$ is generated by the elements of $A$ and the elements of $B$ subject to the relation $\sum_{i=1}^{n} a_{i} b_{i}=0$ if $\sum_{i=1}^{n} a_{i} p b_{i}=0$ (with $a_{i} \in A$ and $b_{i} \in B$ ). This ring $T$ is denoted $A_{(M, p)}^{\sqcup} B$ in [17, Ch. 13]. The maps $\rho_{A}$ and $\rho_{B}$ are obvious and $\rho_{M}$ sends $\sum_{i} a_{i} p b_{i}$ to $\sum_{i} a_{i} b_{i}$.

(2) Suppose $M=A p B \oplus N$ for some $(A, B)$-bimodule $N$. Now $T$ is the tensor ring over $A \underset{(M, p)}{\sqcup} B$ of

$$
(A \underset{(M, p)}{\sqcup} B) \otimes_{A} N \otimes_{B}(A \underset{(M, p)}{\sqcup} B) .
$$

We may vary the choice of $p$ as the following example illustrates:

Example 2.9. Suppose $A=B=M=\mathbb{Z}$ and $p=2$. In this case $T=\mathbb{Z}\left[\frac{1}{2}\right]$ and $\rho_{A}=\rho_{B}$ is the inclusion of $\mathbb{Z}$ in $\mathbb{Z}\left[\frac{1}{2}\right]$ while $\rho_{M}(n)=n / 2$ for all $n \in \mathbb{Z}$.

Example 2.9 can be verified by direct calculation using Theorem 2.4 or deduced from part (1) of Example 2.5 by setting $a_{0}=b_{0}=2$ in the following more general proposition. Before stating it, let us remark that the universal property of $T=$ $T(M, p)$ implies that $T(M, p)$ is functorial in $(M, p)$. An $(A, B)$-bimodule morphism $\phi: M \rightarrow M^{\prime}$ with $\phi(p)=p^{\prime}$ induces a ring morphism $T(M, p) \rightarrow T\left(M^{\prime}, p^{\prime}\right)$.

Proposition 2.10. Suppose $A$ and $B$ are rings, $M$ is an $(A, B)$-bimodule and $p \in M$. If $a_{0} \in A$ and $b_{0} \in B$ satisfy $a_{0} m=m b_{0}$ for all $m \in M$, then:

(1) The element $\rho_{M}\left(a_{0} p\right)=x_{a_{0} p}=x_{p b_{0}}$ is central in $T(M, p)$.

(2) The ring morphism $\phi: T(M, p) \rightarrow T\left(M, a_{0} p\right)=T\left(M, p b_{0}\right)$ induced by the bimodule morphism $\phi: M \rightarrow M ; m \mapsto a_{0} m=m b_{0}$ is the universal localization of $T(M, p)$ making invertible the element $x_{a_{0} p}$.

Since $x_{a_{0} p}$ is central, each element in $T\left(M, a_{0} p\right)$ can be written as a fraction $\alpha / \beta$ with numerator $\alpha \in T(M, p)$ and denominator $\beta=x_{a_{0} p}^{r}$ for some nonnegative integer $r$.

Having described universal localization of the ring $R$ in Theorem 2.4 we may also describe the universal localization $\sigma^{-1} R \otimes_{R} N$ of a left $R$-module $N$.

For the convenience of the reader let us first recall the structure of modules over a triangular matrix ring.

Lemma 2.11. Every left $R$-module $N$ can be written canonically as a triple

$$
\left(N_{A}, N_{B}, f: M \otimes_{B} N_{B} \rightarrow N_{A}\right)
$$

where $N_{A}$ is a left A-module, $N_{B}$ is a left B-module and $f$ is a morphism of left A-modules. 
A proof of this lemma is included in Section 3 below. Localization of modules can be expressed as follows.

Theorem 2.12. 2 For any left $R$-module $N=\left(N_{A}, N_{B}, f\right)$ the localization left $\sigma^{-1} R$-module $\sigma^{-1} N=\sigma^{-1} R \otimes_{R} N$ is isomorphic to $\left(\begin{array}{l}L \\ L\end{array}\right)$ with $\sigma^{-1} R=M_{2}(T)$, $T=T(M, p), L$ the left $T$-module defined by

$$
\begin{aligned}
L & =(T T) \otimes_{R} N \\
& =\operatorname{coker}\left(\left(\begin{array}{c}
1 \otimes f \\
g \otimes 1
\end{array}\right): T \otimes_{A} M \otimes_{B} N_{B} \rightarrow\left(T \otimes_{A} N_{A}\right) \oplus\left(T \otimes_{B} N_{B}\right)\right)
\end{aligned}
$$

with $g$ the $(T, B)$-bimodule morphism

$$
g: T \otimes_{A} M \rightarrow T ; t \otimes m \mapsto-t x_{m},
$$

and $M_{2}(T)$ acting on the left of $\left(\begin{array}{l}L \\ L\end{array}\right)$ by matrix multiplication.

\section{Proofs}

The remainder of this paper is devoted to the proofs of Theorem 2.4. Proposition 2.10 and Theorem 2

3.1. Localization as pushout. Before proving Theorem 2.4 we show that there is a pushout diagram

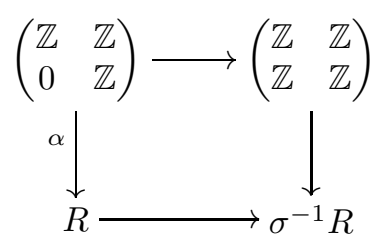

where $\alpha\left(\begin{array}{ll}1 & 0 \\ 0 & 0\end{array}\right)=\left(\begin{array}{ll}1 & 0 \\ 0 & 0\end{array}\right), \alpha\left(\begin{array}{ll}0 & 0 \\ 0 & 1\end{array}\right)=\left(\begin{array}{ll}0 & 0 \\ 0 & 1\end{array}\right)$ and $\alpha\left(\begin{array}{ll}0 & 1 \\ 0 & 0\end{array}\right)=\left(\begin{array}{ll}0 & p \\ 0 & 0\end{array}\right)$. Bergman observed [4, p. 71] that more generally, up to Morita equivalence every localization $R \rightarrow \sigma^{-1} R$ appears in such a pushout diagram.

It suffices to check that the lower horizontal arrow in any pushout

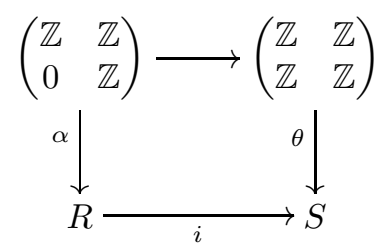

is i) $\sigma$-inverting and ii) universal among $\sigma$-inverting ring morphisms. The universal property of a pushout will be shown to be the universal property of a universal localization, so that such a commutative diagram is a pushout if and only if $S$ is a universal localization $\sigma^{-1} R$.

\footnotetext{
${ }^{2}$ This corrects Theorem 2.12 in the preprint version arXiv:math.RA/0407407.
} 
i) The map id $\otimes \sigma: S \otimes_{R}\left(\begin{array}{c}A \\ 0\end{array}\right) \rightarrow S \otimes_{R}\left(\begin{array}{c}M \\ B\end{array}\right)$ has inverse given by the composite

$$
S \otimes_{R}\left(\begin{array}{c}
M \\
B
\end{array}\right) \subset S \otimes_{R} R \cong S \stackrel{\gamma}{\longrightarrow} S \cong S \otimes_{R} R \rightarrow S \otimes_{R}\left(\begin{array}{c}
A \\
0
\end{array}\right)
$$

where $\gamma$ multiplies on the right by $\theta\left(\begin{array}{ll}0 & 0 \\ 1 & 0\end{array}\right)$.

ii) If $i^{\prime}: R \rightarrow S^{\prime}$ is a $\sigma$-inverting ring morphism, then there is an inverse $\psi$ : $S^{\prime} \otimes_{R}\left(\begin{array}{c}M \\ B\end{array}\right) \rightarrow S^{\prime} \otimes_{R}\left(\begin{array}{c}A \\ 0\end{array}\right)$ to id $\otimes \sigma$. It is argued shortly below that there is a (unique) diagram

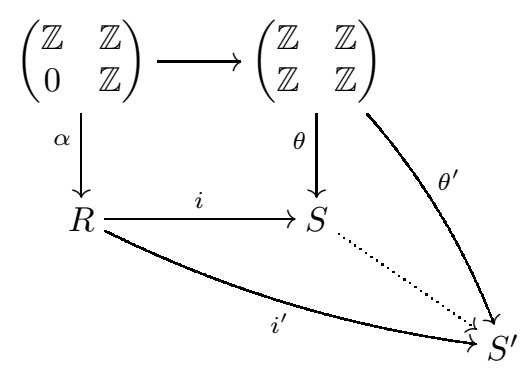

where $\theta^{\prime}$ sends $\left(\begin{array}{ll}0 & 0 \\ 1 & 0\end{array}\right)$ to $\psi\left(1 \otimes\left(\begin{array}{l}0 \\ 1\end{array}\right)\right) \in S^{\prime} \otimes_{R}\left(\begin{array}{l}A \\ 0\end{array}\right) \subset S^{\prime}$. Since $S$ is a pushout, there is a unique morphism $S \rightarrow S^{\prime}$ to complete the diagram and so $i^{\prime}$ factors uniquely through $i$.

To show uniqueness of (1), note that in $S^{\prime}$ multiplication on the right by $\theta^{\prime}\left(\begin{array}{ll}0 & 1 \\ 0 & 0\end{array}\right)$ must coincide with the morphism

$$
\left(\begin{array}{cc}
0 & 0 \\
\mathrm{id} \otimes \sigma & 0
\end{array}\right): S^{\prime} \otimes\left(\begin{array}{c}
A \\
0
\end{array}\right) \oplus S^{\prime} \otimes\left(\begin{array}{c}
M \\
B
\end{array}\right) \longrightarrow S^{\prime} \otimes\left(\begin{array}{c}
A \\
0
\end{array}\right) \oplus S^{\prime} \otimes\left(\begin{array}{c}
M \\
B
\end{array}\right)
$$

so multiplication on the right by $\theta^{\prime}\left(\begin{array}{ll}0 & 0 \\ 1 & 0\end{array}\right)$ coincides with $\left(\begin{array}{ll}0 & \psi \\ 0 & 0\end{array}\right)$. Now $1 \in S^{\prime}$ may be written

$$
\left(1 \otimes\left(\begin{array}{l}
1 \\
0
\end{array}\right), 1 \otimes\left(\begin{array}{l}
0 \\
1
\end{array}\right)\right) \in S^{\prime} \otimes_{R}\left(\begin{array}{l}
A \\
0
\end{array}\right) \oplus S^{\prime} \otimes_{R}\left(\begin{array}{c}
M \\
B
\end{array}\right)
$$

so $\theta^{\prime}\left(\begin{array}{ll}0 & 0 \\ 1 & 0\end{array}\right)=\psi\left(1 \otimes\left(\begin{array}{l}0 \\ 1\end{array}\right)\right)$. The reader may verify that this formula demonstrates the existence of a commutative diagram (11).

3.2. Identifying $\sigma^{-1} R$.

Proof of Theorem 2.4. It suffices to show that the diagram of ring morphisms

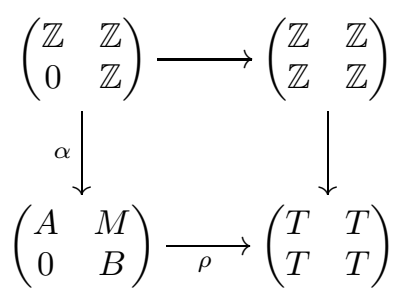


is a pushout, where $T=T(M, p), \rho=\left(\begin{array}{cc}\rho_{A} & \rho_{M} \\ 0 & \rho_{B}\end{array}\right)$ and $\alpha$ is defined as in Section 3.1 Given a diagram of ring morphisms

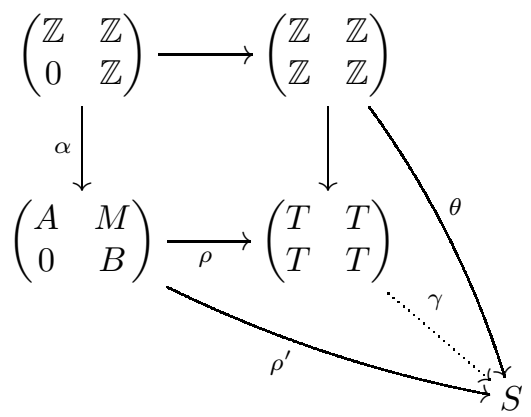

we must show that there is a unique morphism $\gamma$ to complete the diagram. The map $\theta$ induces a decomposition of $S$ as a matrix ring $M_{2}\left(S^{\prime}\right)=\left(\begin{array}{ll}S^{\prime} & S^{\prime} \\ S^{\prime} & S^{\prime}\end{array}\right)$ with $S^{\prime}$ the centralizer of $\theta\left(M_{2}(\mathbb{Z})\right) \subset S$. In particular, $\theta\left(e_{i j}\right)=e_{i j}$ for $i, j \in\{1,2\}$. Any morphism $\gamma$ which makes the diagram commute must be of the form $\gamma=M_{2}\left(\gamma^{\prime}\right)$ for some ring morphism $\gamma^{\prime}: T \rightarrow S^{\prime}$ (e.g. Cohn [9, p. 1] or Lam [15, (17.7)]). Commutativity of the diagram implies that $\rho^{\prime}$ also respects the $2 \times 2$ matrix structure and we may write

$$
\rho^{\prime}=\left(\begin{array}{cc}
\rho_{A}^{\prime} & \rho_{M}^{\prime} \\
0 & \rho_{B}^{\prime}
\end{array}\right):\left(\begin{array}{cc}
A & M \\
0 & B
\end{array}\right) \longrightarrow\left(\begin{array}{cc}
S^{\prime} & S^{\prime} \\
S^{\prime} & S^{\prime}
\end{array}\right)
$$

with $\rho_{M}^{\prime}(p)=1$ as one sees by considering the images of $\left(\begin{array}{ll}0 & 1 \\ 0 & 0\end{array}\right)$ in $\left(\begin{array}{ll}\mathbb{Z} & \mathbb{Z} \\ 0 & \mathbb{Z}\end{array}\right)$ under the maps in diagram (2) above. Since $\rho^{\prime}$ is a ring morphism, one finds

$$
\left(\begin{array}{cc}
\rho_{A}^{\prime}\left(a a^{\prime}\right) & \rho_{M}^{\prime}\left(a m^{\prime}+m b^{\prime}\right) \\
0 & \rho_{B}^{\prime}\left(b b^{\prime}\right)
\end{array}\right)=\left(\begin{array}{cc}
\rho_{A}^{\prime}(a) \rho_{A}^{\prime}\left(a^{\prime}\right) & \rho_{A}^{\prime}(a) \rho_{M}^{\prime}\left(m^{\prime}\right)+\rho_{M}^{\prime}(m) \rho_{B}^{\prime}\left(b^{\prime}\right) \\
0 & \rho_{B}^{\prime}(b) \rho_{B}^{\prime}\left(b^{\prime}\right)
\end{array}\right)
$$

for all $a, a^{\prime} \in A, b, b^{\prime} \in B$ and $m, m^{\prime} \in M$. Hence the maps $\rho_{A}^{\prime}: A \rightarrow S^{\prime}$ and $\rho_{B}^{\prime}: B \rightarrow S^{\prime}$ are ring morphisms and $\rho_{M}^{\prime}$ is a morphism of $(A, B)$-bimodules. Thus $S^{\prime}$ is an $(A, M, B)$-ring with respect to the maps $\rho_{A}^{\prime}, \rho_{M}^{\prime}, \rho_{B}^{\prime}$ such that $\rho_{M}^{\prime}(p)=1$. By the universal property of $T$ there exists a unique morphism $\gamma^{\prime}: T \rightarrow S^{\prime}$ such that $M_{2}\left(\gamma^{\prime}\right): M_{2}(T) \rightarrow M_{2}\left(S^{\prime}\right)=S$ completes diagram (2) above.

Proof of Proposition 2.10, 1. In $T(M, p)$ we have $x_{a_{0} p} x_{m}=x_{a_{0} m}=x_{m b_{0}}=$ $x_{m} x_{p b_{0}}=x_{m} x_{a_{0} p}$ for all $m \in M$.

2. The map $\phi: M \rightarrow M ; m \mapsto a_{0} m$ induces

$$
\begin{aligned}
\phi: T(M, p) & \rightarrow T\left(M, a_{0} p\right), \\
x_{m} & \mapsto x_{a_{0} m} .
\end{aligned}
$$

In particular, $\phi\left(x_{a_{0} p}\right)=x_{a_{0}^{2} p} \in T\left(M, a_{0} p\right)$ and we have

$$
x_{a_{0}^{2} p} x_{p}=x_{a_{0}\left(a_{0} p\right)} x_{p}=x_{a_{0} p}=1=x_{p b_{0}}=x_{p} x_{p b_{0}^{2}}=x_{p} x_{a_{0}^{2} p},
$$

so $\phi\left(x_{a_{0} p}\right)$ is invertible.

We must check that (3) is universal. If $f: T(M, p) \rightarrow S$ is a ring morphism and $f\left(x_{a_{0} p}\right)$ is invertible, we claim that there exists a unique $\widetilde{f}: T\left(M, a_{0} p\right) \rightarrow S$ such that $\widetilde{f} \phi=f$. 
Uniqueness: Suppose $\tilde{f} \phi=f$. For each $m \in M$ we have

$$
\widetilde{f}\left(x_{a_{0} m}\right)=\tilde{f} \phi\left(x_{m}\right)=f\left(x_{m}\right) .
$$

Now $f\left(x_{a_{0} p}\right) \tilde{f}\left(x_{m}\right)=\tilde{f} \phi\left(x_{a_{0} p}\right) \tilde{f}\left(x_{m}\right)=\tilde{f}\left(x_{a_{0}\left(a_{0} p\right)} x_{m}\right)=\widetilde{f}\left(x_{a_{0} m}\right)=f\left(x_{m}\right)$, so

$$
\widetilde{f}\left(x_{m}\right)=\left(f\left(x_{a_{0} p}\right)\right)^{-1} f\left(x_{m}\right) .
$$

Existence: It is straightforward to check that equation (4) provides a definition of $\widetilde{f}$ which respects the relations (+), (a), (b) and (id) in $T\left(M, a_{0} p\right)$. Relation (b), for example, is proved by the equations

$$
\widetilde{f}\left(x_{m}\right) \widetilde{f}\left(x_{a_{0} p b}\right)=f\left(x_{a_{0} p}\right)^{-1} f\left(x_{m}\right) f\left(x_{p b}\right)=f\left(x_{a_{0} p}\right)^{-1} f\left(x_{m b}\right)=\widetilde{f}\left(x_{m b}\right)
$$

and the other relations are left to the reader.

3.3. Module localization. We turn finally to the universal localization $\sigma^{-1} R \otimes_{R}$ $N$ of an $R$-module $N$.

Proof of Lemma 2.11. If $N$ is a left $R$-module, set $N_{A}=\left(\begin{array}{ll}1 & 0 \\ 0 & 0\end{array}\right) N$ and set $N_{B}=$ $N / N_{A}$. If $m \in M$ and $n_{B} \in N_{B}$ choose a lift $x \in N$ and define the map $f$ : $M \otimes N_{B} \rightarrow N_{A}$ by $f\left(m \otimes n_{B}\right)=\left(\begin{array}{cc}0 & m \\ 0 & 0\end{array}\right) x$. Conversely, given a triple $\left(N_{A}, N_{B}, f\right)$ one recovers a left $R$-module $\left(\begin{array}{c}N_{A} \\ N_{B}\end{array}\right)$ with

$$
\left(\begin{array}{cc}
a & m \\
0 & b
\end{array}\right)\left(\begin{array}{l}
n_{A} \\
n_{B}
\end{array}\right)=\left(\begin{array}{c}
a n_{A}+f\left(m \otimes n_{B}\right) \\
b n_{B}
\end{array}\right)
$$

for all $a \in A, b \in B, m \in M, n_{A} \in N_{A}, n_{B} \in N_{B}$.

Proof of Theorem 2. As in the statement, let $T=T(M, p)$ and define the left $T$ module

$$
L=\operatorname{coker}\left(\left(\begin{array}{c}
1 \otimes f \\
g \otimes 1
\end{array}\right): T \otimes_{A} M \otimes_{B} N_{B} \rightarrow\left(T \otimes_{A} N_{A}\right) \oplus\left(T \otimes_{B} N_{B}\right)\right) .
$$

We shall establish an isomorphism of left $T$-modules

$$
\left(\begin{array}{ll}
T & T
\end{array}\right) \otimes_{R}\left(\begin{array}{l}
N_{A} \\
N_{B}
\end{array}\right) \cong L
$$

and leave to the reader the straightforward deduction that there is an isomorphism of $\sigma^{-1} R$-modules

$$
\sigma^{-1} R \otimes_{R} N=\left(\begin{array}{cc}
T & T \\
T & T
\end{array}\right) \otimes_{R}\left(\begin{array}{c}
N_{A} \\
N_{B}
\end{array}\right) \cong\left(\begin{array}{l}
L \\
L
\end{array}\right)
$$

The left $T$-module morphism

$$
\begin{aligned}
& \alpha: L \rightarrow\left(\begin{array}{ll}
T & T
\end{array}\right) \otimes_{R}\left(\begin{array}{l}
N_{A} \\
N_{B}
\end{array}\right) ; \\
& \left(t \otimes n_{A}, t^{\prime} \otimes n_{B}\right) \mapsto\left(\begin{array}{ll}
t & 0
\end{array}\right) \otimes_{R}\left(\begin{array}{c}
n_{A} \\
0
\end{array}\right)+\left(\begin{array}{ll}
0 & t^{\prime}
\end{array}\right) \otimes_{R}\left(\begin{array}{c}
0 \\
n_{B}
\end{array}\right)
\end{aligned}
$$


is well defined, since

$$
\begin{aligned}
\alpha\left(t \otimes_{A} f\left(m, n_{B}\right), g(t, m) \otimes_{B} n_{B}\right) & =\alpha\left(t \otimes_{A} f\left(m, n_{B}\right),-t x_{m} \otimes_{B} n_{B}\right) \\
& =\left(\begin{array}{ll}
t & 0
\end{array}\right) \otimes_{R}\left(\begin{array}{c}
f\left(m, n_{B}\right) \\
0
\end{array}\right)-\left(\begin{array}{ll}
0 & t x_{m}
\end{array}\right) \otimes_{R}\left(\begin{array}{c}
0 \\
n_{B}
\end{array}\right) \\
& =\left(\begin{array}{ll}
t & 0
\end{array}\right) \otimes_{R}\left(\begin{array}{cc}
0 & m \\
0 & 0
\end{array}\right)\left(\begin{array}{c}
0 \\
n_{B}
\end{array}\right)-\left(\begin{array}{cc}
t & 0
\end{array}\right)\left(\begin{array}{cc}
0 & m \\
0 & 0
\end{array}\right) \otimes_{R}\left(\begin{array}{c}
0 \\
n_{B}
\end{array}\right) \\
& =0 \in\left(\begin{array}{ll}
T & T
\end{array}\right) \otimes_{R}\left(\begin{array}{c}
N_{A} \\
N_{B}
\end{array}\right) .
\end{aligned}
$$

The left $T$-module morphism

$$
\beta:\left(\begin{array}{ll}
T & T
\end{array}\right) \otimes_{R}\left(\begin{array}{l}
N_{A} \\
N_{B}
\end{array}\right) \rightarrow L ;\left(\begin{array}{ll}
t & t^{\prime}
\end{array}\right) \otimes_{R}\left(\begin{array}{c}
n_{A} \\
n_{B}
\end{array}\right) \mapsto\left(t \otimes n_{A}, t^{\prime} \otimes n_{B}\right)
$$

is well defined, since

$$
\begin{aligned}
\beta\left(\left(\begin{array}{ll}
t & t^{\prime}
\end{array}\right) \otimes_{R}\left(\begin{array}{cc}
a & m \\
0 & b
\end{array}\right)\left(\begin{array}{c}
n_{A} \\
n_{B}
\end{array}\right)-\left(\begin{array}{ll}
t & t^{\prime}
\end{array}\right)\left(\begin{array}{cc}
a & m \\
0 & b
\end{array}\right) \otimes_{R}\left(\begin{array}{c}
n_{A} \\
n_{B}
\end{array}\right)\right) \\
=\left(t \otimes\left(a n_{A}+f\left(m, n_{b}\right)\right), t^{\prime} \otimes b n_{B}\right)-\left(t a \otimes n_{A},\left(t x_{m}+t^{\prime} b\right) \otimes n_{B}\right) \\
=\left(t \otimes f\left(m, n_{B}\right),-t x_{m} \otimes n_{B}\right) \\
=(1 \otimes f, g \otimes 1)\left(t \otimes m \otimes n_{B}\right)=0 \in L .
\end{aligned}
$$

It is immediate that $\beta \alpha=\mathrm{id}$. To prove (5) we must check that $\alpha \beta=\mathrm{id}$ or in other words, that

$$
\left(\begin{array}{ll}
t & t^{\prime}
\end{array}\right) \otimes_{R}\left(\begin{array}{c}
n_{A} \\
n_{B}
\end{array}\right)=\left(\begin{array}{ll}
t & 0
\end{array}\right) \otimes_{R}\left(\begin{array}{c}
n_{A} \\
0
\end{array}\right)+\left(\begin{array}{ll}
0 & t^{\prime}
\end{array}\right) \otimes_{R}\left(\begin{array}{c}
0 \\
n_{B}
\end{array}\right)
$$

This equation follows from the next two calculations:

$$
\begin{aligned}
& \left(\begin{array}{ll}
t & 0
\end{array}\right) \otimes_{R}\left(\begin{array}{c}
0 \\
n_{B}
\end{array}\right)=\left(\begin{array}{ll}
t & 0
\end{array}\right)\left(\begin{array}{ll}
0 & 0 \\
0 & 1
\end{array}\right) \otimes_{R}\left(\begin{array}{c}
0 \\
n_{B}
\end{array}\right)=0 ; \\
& \left(\begin{array}{ll}
0 & t^{\prime}
\end{array}\right) \otimes_{R}\left(\begin{array}{c}
n_{A} \\
0
\end{array}\right)=\left(\begin{array}{ll}
0 & t^{\prime}
\end{array}\right)\left(\begin{array}{ll}
1 & 0 \\
0 & 0
\end{array}\right) \otimes_{R}\left(\begin{array}{c}
n_{A} \\
0
\end{array}\right)=0 .
\end{aligned}
$$

\section{REFERENCES}

[1] P. Ara, M. A. González-Barroso, K. R. Goodearl, and E. Pardo. Fractional skew monoid rings. Journal of Algebra, 278(1):104-126, 2004. MR2068068 (2005f:16042)

[2] D. J. Benson. Representations and cohomology. I. Basic Representation Theory of finite groups and associative algebras. Cambridge Studies in Advanced Mathematics, 30. Cambridge University Press, 1995. MR.1110581 (92m:20005)

[3] G. M. Bergman. Modules over coproducts of rings. Transactions of the American Mathematical Society, 200:1-32, 1974. MR0357502 (50:9970)

[4] G. M. Bergman. Coproducts and some universal ring constructions. Transactions of the American Mathematical Society, 200:33-88, 1974. MR0357503(50:9971)

[5] G. M. Bergman and W. Dicks. Universal derivations and universal ring constructions. Pacific Journal of Mathematics, 79(2):293-337, 1978. MR0531320 (81b:16024) 
[6] P. M. Cohn. Localization in general rings, a historical survey. Proceedings of the Conference on Noncommutative Localization in Algebra and Topology, ICMS, Edinburgh, 29-30 April, 2002, London Mathematical Society Lecture Notes 330, Cambridge University Press, 5-23, 2006.

[7] P. M. Cohn. Free Rings and their Relations. London Mathematical Society Monographs, 2. Academic Press, London, 1971. MR0371938 (51:8155)

[8] P. M. Cohn. Rings of fractions. American Mathematical Monthly, 78:596-615, 1971. MR0285561 (44:2779)

[9] P. M. Cohn. Free Rings and their Relations. London Mathematical Society Monographs, 19. Academic Press, London, second edition, 1985. MR0800091 (87e:16006)

[10] P.M. Cohn and W. Dicks. Localization in semifirs. II. J. London Math.Soc. (2), 13(3):411418, 1976. MR0424856 (54:12814)

[11] W. Dicks and E. Sontag. Sylvester domains. J. Pure Appl. Algebra, 13(3):243-275, 1978. MR.0509164 (80j:16014)

[12] M. Farber and P. Vogel. The Cohn localization of the free group ring. Mathematical Proceedings of the Cambridge Philosophical Society, 111(3):433-443, 1992. MR1151322 (93b:16049)

[13] A. Haghany and K. Varadarajan. Study of formal triangular matrix rings. Communications in Algebra, 27(11):5507-5525, 1999. MR 1713049 (2000g:16003)

[14] A. Haghany and K. Varadarajan. Study of modules over formal triangular matrix rings. Journal of Pure and Applied Algebra, 147(1):41-58, 2000. MR1744654 (2000k:16004)

[15] T. Y. Lam. Lectures on Modules and Rings. Number 189 in Graduate Texts in Mathematics. Springer, New York, 1999. MR1653294 (99i:16001)

[16] A. A. Ranicki. Noncommutative localization in topology. Proceedings of the Conference on Noncommutative Localization in Algebra and Topology, ICMS, Edinburgh, 29-30 April, 2002. arXiv:math.AT/0303046, London Mathematical Society Lecture Notes 330, Cambridge University Press, 81-102, 2006.

[17] A. H. Schofield. Representations of rings over skew fields, Volume 92 of London Mathematical Society Lecture Note Series. Cambridge University Press, 1985. MR0800853 (87c:16001)

Department of Mathematics, International University Bremen, Bremen 28759, GerMANY 